\title{
What does a phytophysiognomic mosaic reveal about mosses and liverworts from the subtropical Atlantic Forest?
}

\author{
Talita da Silva Dewes ${ }^{1 *}$ (1), Nivea Dias dos Santos ${ }^{2}$ (I) and Juçara Bordin ${ }^{1,3}$ (1)
}

Received: September 19, 2020

Accepted: December 19, 2020

\begin{abstract}
The Atlantic Forest contains more bryophyte species and greater endemism than any other Brazilian phytogeographic domain. We analyzed the bryoflora of an Atlantic Forest remnant along the southern Brazilian coast to examine: (i) floristic composition, species richness, life forms and bryocenological groups in different phytophysiognomies (humid lowlands, sandy Restinga forests, and swampy Restinga forests); (ii) floristic similarities among them; and, (iii) the influence of substrate types on bryophyte species richness and floristic composition. Sandy Restinga forest had the greatest species richness. Approximately $75 \%$ of the species and $42 \%$ of the families were not shared among phytophysiognomies. The predominant life form was mat (46 \%) and the predominant bryocenological group was corticolous (69\%). Elevated floristic differentiation was observed among the different phytophysiognomies (Jaccard values $<0.35)$ and among the different substrates sampled $(<0.2)$. The floristic uniqueness found among the different physiognomies in the present study demonstrates the importance of studying the diversity and conservation of all Atlantic Forest phytophysiognomies. The evaluation of bryophytes to delimit and manage protected areas can contribute to effective protection of the entire biodiversity spectrum. In addition to their important ecological roles, bryophytes are useful bio-indicators and valuable tools for monitoring, conserving, and restoring ecosystems.
\end{abstract}

Keywords: Bryophytes, Atlantic Forest domain, Itapeva State Park, pioneer formations, sandy Restinga forest, swampy Restinga forest

\section{Introduction}

Many of the most exploited and threatened natural ecosystems in the world occur in coastal areas (Worm et al. 2006). Those areas suffer strong anthropic impacts due to urban expansion, sand mining, wetland drainage, vegetation trampling, vehicle traffic, the introduction of exotic species, and the discarding of solid residues and liquid effluents (Waechter 1985; Brack 2009). Those aggressions have caused enormous biodiversity losses and the disruption of ecosystem functions that contribute to biological invasions, diminished water quality, and diminished coastal protection against flooding events and storms (Koch et al. 2009). The Atlantic Forest is considered a global biodiversity hotspot because of its high level of endemism, although intense degradation has significantly reduced its original extension along the Brazilian coast (Mittermeier et al. 2004).

Atlantic Forest vegetation occurs along the entire eastern coast of Brazil, from Rio Grande do Sul State in the south and to Rio Grande do Norte State in the north, and extends inland to the states of Minas Gerais, Mato

1 Universidade Estadual do Rio Grande do Sul, 95400-000, São Francisco de Paula,RS, Brazil

2 Departamento de Botânica, Instituto de Ciências Biológicas e da Saúde, Universidade Federal Rural do Rio de Janeiro, 23897-000,

Seropédica, RJ, Brazil

3 Universidade Estadual do Rio Grande do Sul, 95520-000, Osório, RS, Brazil

* Corresponding author: talitadewes@gmail.com.br 
Grosso do Sul, and Goiás. The Atlantic Forest is characterized by formations that principally include Dense, Open, and Mixed Ombrophilous Forests, as well as Semi-Deciduous, Deciduous, and Evergreen Seasonal Forests - which originally represented approximately $85 \%$ of the cover of that phytogeographic domain (IBGE 2019).

The coast of Rio Grande do Sul State extends for approximately $620 \mathrm{~km}$, with an extensive inland plain dating to the Quaternary period (Buchmann et al. 2009). Outcroppings of basaltic rock of the Serra Geral and sandstones of the Botucatu formation are found exclusively in the municipality of Torres along the extreme northern coast of the state, and are geologically more ancient than the Coastal Plain, with its sandy landscape and modified plant cover (Waechter 1985; Duarte \& Bencke 2006; Buchmann et al. 2009). That varied geomorphology, together with climatic differences, result in a mosaic of phytophysiognomies there different from typical Atlantic Forest formations.

The coastal plain of Rio Grande do Sul State is dominated by vegetation formations showing both marine and fluviomarine influences (considered "pioneer formations"), which, north of the $30^{\circ}$ parallel, are in contact with Ombrophilous Forest formations of the Atlantic Forest domain (Waechter 1985; IBGE 2019). Climatic and edaphic conditions there, together with high humidity and salinity, support a mosaic of phytophysiognomies that include frontal dune vegetation, "buriti" palm forests, open fields and forests occupying sandy soils characteristic of dryland areas, as well as "banhados", "juncais", "maricazais", "sarandizais", flooded fields, and humid forests characteristic of wet soils (swampy Restinga forests) (Waechter 1985; IBGE 2012).

The Atlantic Forest has the largest number of species and the greatest endemism of bryophytes of any other Brazilian phytogeographic domain, and likewise demonstrates the largest variety of life forms and substrates colonized by those plants (Costa \& Peralta 2015). Different from vascular plants, which demonstrate their greatest richness near the equator, bryophyte species richness is directly related to environmental heterogeneity, independent of their location on the globe (Hedenäs 2007; Shaw et al. 2005; Möls et al. 2013). According to Glime (2017a), cryptogams, especially lichens and bryophytes, are among the most sensitive plants for indicating atmospheric pollution and environmental disturbances. The roles that bryophytes have taken on as bioindicators reflect their poikilohydric natures, that is, their gametophytes demonstrate high permeability to water and other chemical substances (Proctor 1979). They are therefore directly exposed to the external environment and react quickly to environmental variations, demonstrating alterations that range from intracellular to community level disturbances (Gradstein et al. 2001; Vanderpooten \& Goffinet 2009).

More than 300 publications have reported using bryophytes directly or indirectly as bioindicators (Glime 2017a). Studies developed in the last five years in Brazil have addressed the relationships of different ecological aspects of bryophytes with microclimatic conditions (Löbs et al. 2019), successional stages (Takashima-Oliveira et al. 2020), phytophysiognomies (Cerqueira et al. 2017), vertical zonation (Oliveira \& Oliveira 2016), and environmental gradients (Souza et al. 2020). The variation of species along environmental gradients, including those of different phytophysiognomies, is an important source of diversity, reflects the environmental diversity within tropical forests (Hedenäs 2007; Shaw et al. 2005), and represents an important aspect to be considered when evaluating the conservation status of a region and its biodiversity preservation (Condit et al. 2002; Mutke \& Barthlott 2005).

Considering the potential of bryophytes as bioindicators, the relevance of the Itapeva State Park (PEVA) reserve for protecting the coastal zone of Rio Grande do Sul State from urban expansion and environmental degradation, and the importance of conserving a remnant mosaic of Atlantic Forest phytophysiognomies, the present study examined the richness of bryophyte species as well as their distributions on different substrates and in different phytophysiognomies (humid lowland areas, sandy and swampy Restinga forests) of the PEVA. To reach those objectives, the following questions oriented our work: (i) what are the floristic compositions and species richness of the different phytophysiognomies, and what life forms and bryocenological groups are present in each?; (ii) what are the floristic similarities between the different phytophysiognomies?; and, (iii) what is the influence of substrate type on bryophyte species richness and floristic composition?

\section{Materials and methods}

\section{Study area}

The Itapeva State Park is an integral protection conservation area created in 2002 (Rio Grande do Sul 2002) and part of the Brazilian National System of Conservation Areas - SNUC (in Portuguese Sistema Nacional de Unidades de Conservação da Natureza). The park is situated in the municipality of Torres ( $29^{\circ} 21^{\prime} 51^{\prime \prime} \mathrm{S}$ x $49^{\circ} 45^{\prime} 03^{\prime \prime} \mathrm{W}$ ), on the northern coast of Rio Grande do Sul State, between Balneário Itapeva to the south, Estrada do Mar (RS-389) to the west, the Atlantic Ocean to the east, and the urban area of Torres to the north. The park covers approximately $1000 \mathrm{ha}$, and its buffer zone surpasses a radius of $10 \mathrm{~km}$ (Duarte \& Bencke 2006).

The PEVA was created to preserve natural ecosystems with significant ecological relevance and high scenic beauty. The park also encourages scientific research, environmental education activities, recreation in contact with nature, and ecotourism (Duarte \& Bencke 2006). The natural ecosystems within the PEVA integrate the Atlantic Forest phytogeographic domain, with diverse 
phytophysiognomies. Those natural phytophysiognomies include pioneer herbaceous formations (classified as halophytic, psamophyllous, and limnophylous) that occupy the frontal dunes, mobile interior dunes/humid fields and swamplands ("banhados"), as well as forest formations classified as "Sandy Restinga" (growing on dunes or rock outcrops) and "Swampy Restinga forest". The PEVA also contains a shrub-arboreal formation with the significant presence of Butia capitata (Mart.) Becc. palms (called "butiazal" formations) (Duarte \& Bencke 2006). With the exception of forested areas on the low mountains associated with the Serra Geral basaltic formations and the sandstones of the Botucatu Formation, the other phytophysiognomies occur on a geomorphological formation denominated (in an ample phytogeographic sense) "Restinga" (Duarte \& Bencke 2006; Brack 2009; Buchmann et al. 2009). The regional climate is subtropical humid maritime, with a strong maritime influence conditioned by the local landscape and characterized by mild austral summers and winters (type $\mathrm{Cfa}$, according to the Köppen classification) (Duarte $\&$ Bencke 2006). The mean annual temperature there is 18.9 ${ }^{\circ} \mathrm{C}$ and precipitation $1400 \mathrm{~mm}$ (Ferraro \& Hasenack 2009).

The identifications of the phytophysiognomies selected for the present study were adapted from Waechter (1985) and Duarte \& Bencke (2006): humid lowlands (HL); sandy Restinga forests growing on dunes (SRFD), sandy Restinga forests growing on rock outcrops (SRFR), and swampy Restinga forests (SWRF) (Fig. 1). The HL areas are located between the frontal dunes and the mobile interior dunes, and harbor a psamophilous herbaceous vegetation adapted to sandy, humid soils. The SRFD forests are also associated with sandy soils (although well-drained), and are principally located on the tops and internal flanks of mobile interior dunes; their vegetations are composed of shrubs and small trees as well as phanerogams (principally represented by the families Asteraceae, Anacardiaceae, Myrsinaceae, Cactaceae, and Verbenaceae). The SRFR forests grow on rock outcrops at elevations of approximately $60 \mathrm{~m}$ a.s.l.; their floristic
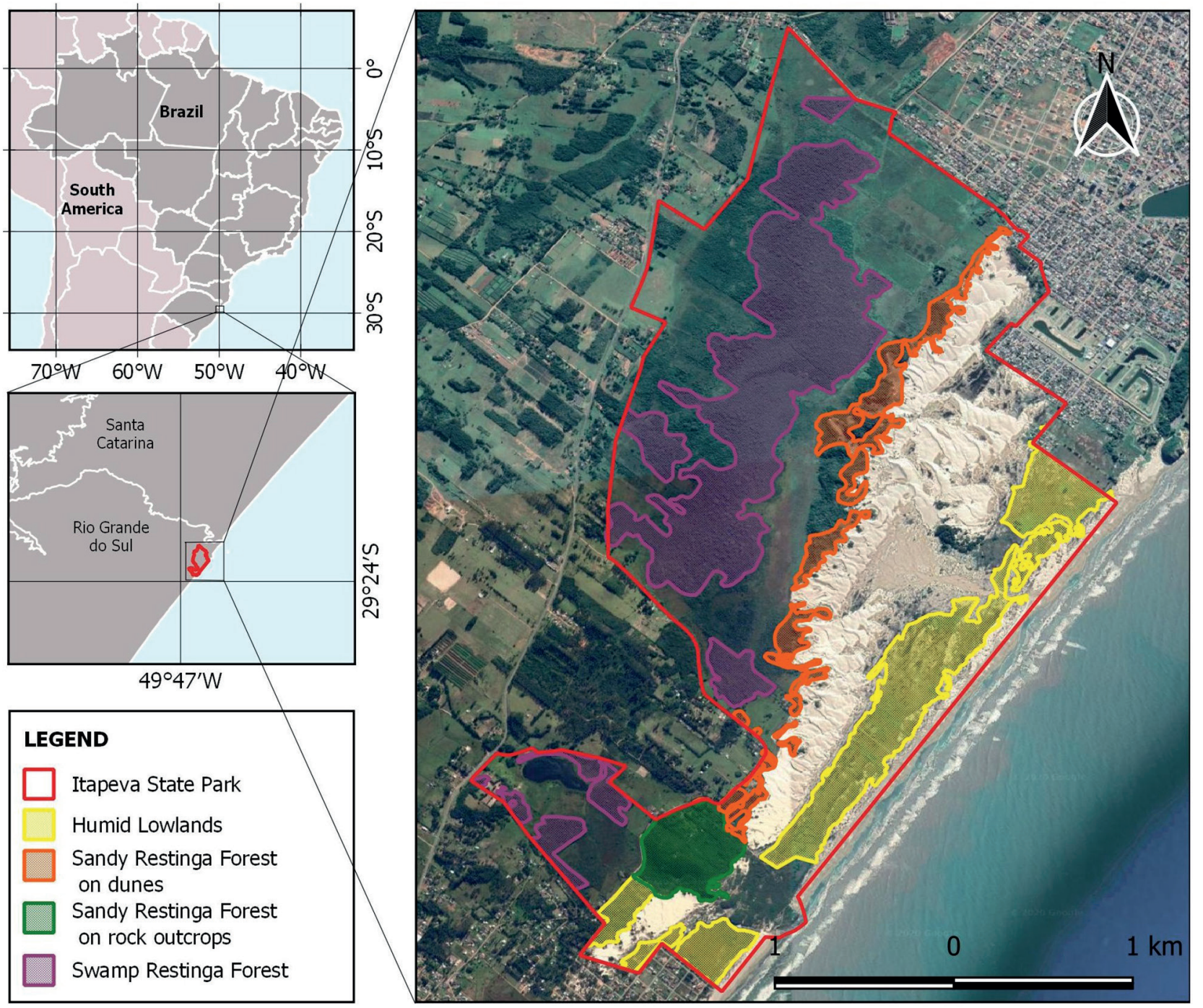

Figure 1. Localization and phytophysiognomies in the Itavepa State Park, Torres, Rio Grande do Sul, Brazil. Source: adapted from Duarte \& Bencke (2006). 
composition consists of vascular plants similar to those of the Dense Ombrophilous Forest (principally represented by families Sapindaceae, Rubiaceae, Euphorbiaceae, Myrtaceae, and Aquifoliaceae). There is a trail through the SRFR forest that we considered separately (and designated as SRFR-T) used for environmental education activities within the PEVA; the interior of the forest ( $2 \mathrm{~m}$ from the trail) is designated as SRFR-I. The SWRF forests grow on poorly-drained and turf soils in a humid microclimate with relatively stable temperatures. That tropical vegetation is notably rich in epiphytes (principally represented by the families Orchidaceae, Bromeliaceae, Araceae, Arecaceae, Heliconiaceae, Clusiaceae, Moraceae, Cecropiaceae, Marcgraviaceae, and Gesneriaceae) (Waechter 1985; Duarte \& Bencke 2006).

\section{Sampling and study material}

Sampling was performed during random walks through the vegetation (Filgueiras et al. 1994), using the common collection method, following Frahm (2003). The specimens collected in SRFR-I and SRFR-T were considered separately in some of the analyses in order to compare their floristic compositions. The mean times spent on collections in each phytophysiognomy were: three hours in HL, SRFR-I, SRFR-T, and SWRF, and four hours in SRFD (due to the difficulty of locomotion there). All colonized substrates were sampled, including tree trunks, soil, rocks, and other surfaces, with collections from trees reaching up to ca. $2 \mathrm{~m}$ above ground level.

The morphological analyses of the species were undertaken in the Laboratório de Biologia e Conservação at UERGS - Litoral Norte (LABeC), using stereo- and optical microscopes. Identifications were based on the specialized literature, examinations of herbarium material, and consultations with specialists. The taxonomic classifications of the species follow the system of Crandall-Stotler et al. (2009) for the Marchantiophyta, and Goffinet et al. (2009) for the Bryophyta. The specimens were subsequently incorporated into the Dr. Ronaldo Wasum Herbarium collections at the Universidade Estadual do Rio Grande do Sul - Litoral Norte (HERW).

In order to verify the influence of substrate type on bryophyte species richness and floristic composition, the specimens were classified into the following bryocenological groups (modified from Gams (1932) and Fudali (2001)): arenicolous (AR - sandy sediments); chasmophytes (CA - artificial substrates); corticolous (CO - live tree trunks); epiphyllous (EP - leaves); epixylic (EX - dead tree trunks); terricolous (TE - soils); and saxicolous (SA - rocks). The life forms were classified as dendroid (D) or mat (M), with the latter differentiated as thalloid mat (Th) (as they have different shapes and retain moisture differently), pendant (P), turf (T), or weft (W), adapted from Mägdefrau (1982).

\section{Data analysis}

Two data matrices were elaborated: (i) species $x$ phytophysiognomies, and (ii) species $x$ substrates, considering the species as descriptors and the phytophysiognomies/ substrates as samples. Initially, an outlier analysis was performed using the Jaccard index with a cut-off level of 2.0 in the PCOrd program (McCunne \& Mefford 1999) to identify possible samples (phytophysiognomies or substrates) having species compositions notably distinct from the general data set. No sample demonstrated a standard error $>2.0$ in the two matrices evaluated. The variations of floristic composition between the phytophysiognomies and substrate types were evaluated using the Unweighted Pair Group Method with Arithmetic Mean (UPGMA), utilizing the Jaccard similarity coefficient, and ordination analysis (Correspondence Analysis - CA), run on Fitopac 2.1 software (Shepherd 2010).

\section{Results}

\section{Species richness}

We encountered 98 bryophyte species in the PEVA (Tab. 1). The phytophysiognomies having the greatest species richness were SRFR (67 spp., being 35 mosses and 32 liverworts) and SWRF (39 spp., 20 mosses and 19 liverworts), while SRFD (11 spp., eight mosses and three liverworts) and HL (nine spp., six mosses and three liverworts) had significantly few taxa (Fig. 2A).

Those species belonged to 31 families ( 21 moss and 10 liverwort). Of the 24 families encountered in SRFR, those with the largest numbers of species were Lejeuneaceae (14 species), Metzgeriaceae (seven), Fissidentaceae (six), and Plagiochilaceae (six); 18 families occurred in SWRF, with the richest being Lejeuneaceae (10 species) and Orthotrichaceae (four). In SRFD, the species distributions were more equitable, as among the nine families identified only Lejeuneaceae and Bryaceae had more than one representative, being two species of Lejeunea and two of Bryum; six families occurred in HL, with Dicranaceae (three species) and Cephaloziellaceae (two) being the most species rich.

\section{Life forms}

The bryophytes of the PEVA are distributed among six life forms (Tab. 1). The mat life form predominates (46\%), including thalloid taxa. The mat forms are represented principally by liverworts belonging to the families Lejeuneaceae, Frullaniaceae, and Metzgeriaceae; it predominates in the two portions of the SRFR (SRFR-I, $55 \%$ and SRFR-T, $42 \%$ ), and also in SWRF (54\%). Dendroid and pendant life forms were encountered only in SRFR-T (24\%), SRFR-I (29\%), and SWRF (18\%). More types of life forms occur in those phytophysiognomies than seen in SRFD or HL. The predominant life forms in SRFD and HL were turf (36\% and $56 \%$ ) and weft (27\% and $22 \%$ ) (Fig. 2B). 


\section{Floristic composition by phytophysiognomy}

Just as species richness is not distributed uniformly in the phytophysiognomies of the PEVA, the floristic compositions of the areas were likewise heterogeneous. Approximately $42 \%$ of the bryophytes families were encountered exclusively in a single phytophysiognomy. Seven families were encountered only in the SRFR (Aneuraceae, Fabroniaceae, Hypopterygiaceae, Lophocoleaceae, Meteoriaceae, Pilotrichaceae, and Rachitheciaceae), four only in SWRF (Cryphaeaceae, Lepidoziaceae, Phyllogoniaceae, and Radulaceae), and two only in HL (Cephaloziellaceae and Pallaviciniaceae). No family was exclusive to the SRFD.

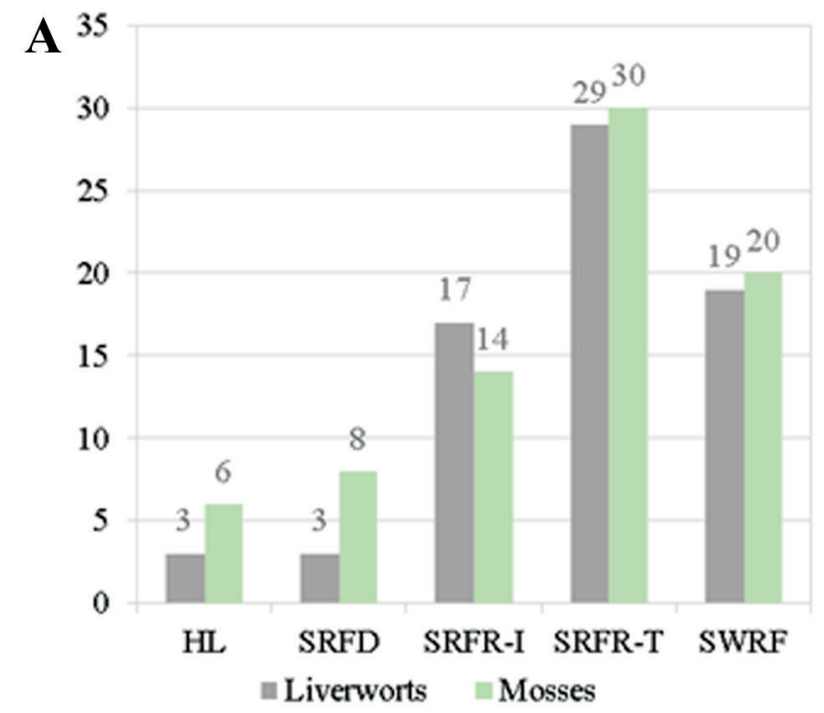

None of the species were encountered in all of the phytophysiognomies, and only four were present in three of them (remembering that SRFR-I and SRFR-T are separated in Figure 2C, but represent a single phytophysiognomy). The species Helicodontium capillare, Macromitrium richardii, and Brittonodoxa subpinnata were present in SRFD, SWRF and SRFR. The species Isopterygium tenerum was present in HL, SWRF and SRFR.

As can be seen in Figure 2C, approximately $75 \%$ of the species were exclusive to only a single phytophysiognomy. In proportional terms of species richness, SRFR demonstrated the greatest exclusivity of species (45), corresponding to $67 \%$ of the bryophytes identified in that phytophysiognomy.

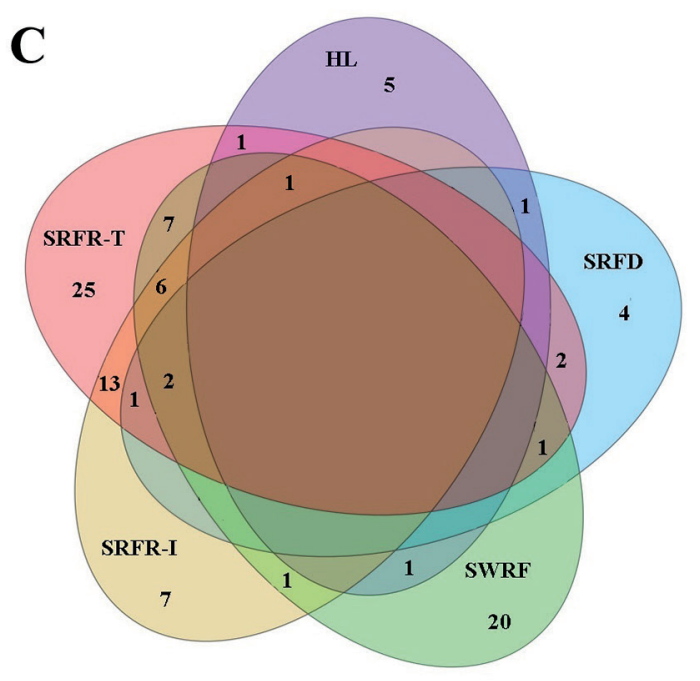

B

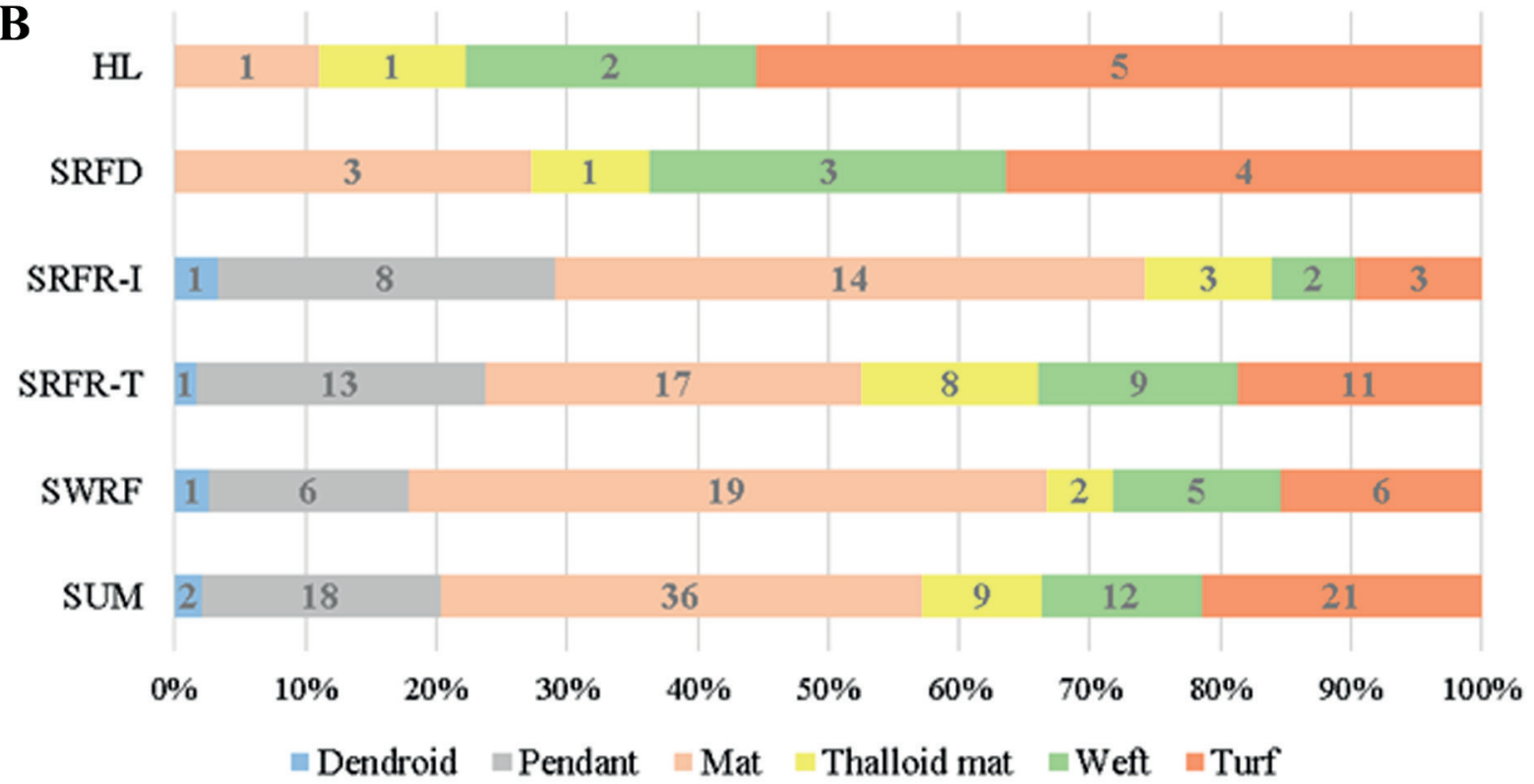

Figure 2. Classification of bryophyte species in the different phytophysiognomies in the Itapeva State Park, Torres, Rio Grande do Sul State, Brazil. A. Species richness by division. B. Life forms of species. C. Venn diagram, demonstrating the numbers of bryophyte species common to, exclusive to, and total in the different phytophysiognomies. HL= humid lowlands. SRFD= sandy Restinga forest growing on dunes. SWRF= swampy Restinga forest. SRFR-I= sandy Restinga forest growing on rock outcrops (interior). SRFR-T= sandy Restinga forest growing on rock outcrops (trail). 
Table 1. List of bryophyte species and their frequencies of occurrence in the different phytophysiognomies and on different substrates in the Itapeva State Park, Torres, Rio Grande do Sul State, Brazil. HL= humid lowlands. SRFD= sandy Restinga forest growing on dunes. SWRF= swampy Restinga forest. SRFR-I= sandy Restinga forest growing on rock outcrops (interior). SRFR-T= sandy Restinga forest growing on rock outcrops (trail). $\mathrm{AR}=$ arenicolous. $\mathrm{CA}=$ chasmophyte. $\mathrm{CO}=$ corticolous. $\mathrm{EP}=$ epiphyllous. $\mathrm{EX}=$ epixylic. $\mathrm{SA}=$ saxicolous. $\mathrm{TE}=$ terricolous. $\mathrm{D}=$ dendroid. $\mathrm{M}=$ mat. $\mathrm{Th}=$ thalloid mat. $\mathrm{P}=$ pendant. $\mathrm{T}=$ turf. $\mathrm{W}=$ weft. ${ }^{*}$ Species exclusive to a single phytophysiognomy.

\begin{tabular}{|c|c|c|c|c|c|c|c|c|c|c|c|c|c|c|}
\hline \multirow[b]{2}{*}{ Taxon } & \multirow{2}{*}{$\frac{\Xi}{\frac{\Xi}{0}}$} & \multicolumn{5}{|c|}{ Phytophysiognomies } & \multicolumn{7}{|c|}{ Bryocenological groups } & \multirow[b]{2}{*}{$\begin{array}{c}\text { Abbreviation of the } \\
\text { taxon }\end{array}$} \\
\hline & & 로 & 星 & 誉 & 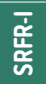 & 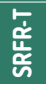 & $\stackrel{\alpha}{<}$ & త & 8 & 욥 & x & $\overleftarrow{n}$ & $\ddot{⺊}$ & \\
\hline \multicolumn{15}{|l|}{ BRYOPHYTA } \\
\hline \multicolumn{15}{|l|}{ Brachytheciaceae } \\
\hline Aerolindigia capilacea (Hornsch.) M. Menzel* & $\mathrm{P}$ & 0 & 0 & 0 & 0 & 1 & 0 & 0 & 1 & 0 & 0 & 0 & 0 & Aer cap \\
\hline Helicodontium capillare (Hedw.) A. Jaeger & W & 0 & 6 & 7 & 8 & 8 & 1 & 0 & 23 & 0 & 3 & 1 & 1 & Hel cap \\
\hline Rhynchostegium scariosum (Taylor) A. Jaeger* & W & 0 & 0 & 0 & 0 & 3 & 0 & 0 & 2 & 0 & 1 & 0 & 0 & Rhy sca \\
\hline Rhynchostegium serrulatum (Hedw.) A. Jaeger* & W & 0 & 0 & 0 & 1 & 4 & 0 & 0 & 0 & 0 & 5 & 0 & 0 & Rhy ser \\
\hline \multicolumn{15}{|l|}{ Bryaceae } \\
\hline Bryum argenteum Broth* & $\mathrm{T}$ & 0 & 1 & 0 & 0 & 0 & 1 & 0 & 0 & 0 & 0 & 0 & 0 & Bry arg \\
\hline Bryum atenense Williams* & $\mathrm{T}$ & 0 & 1 & 0 & 0 & 0 & 1 & 0 & 0 & 0 & 0 & 0 & 0 & Bry ate \\
\hline Bryum coronatum Schwägr.* & $\mathrm{T}$ & 1 & 0 & 0 & 0 & 0 & 0 & 0 & 0 & 0 & 0 & 0 & 1 & Bry cor \\
\hline Rosulabryum densifolium (Brid.) Ochyra* & $\mathrm{T}$ & 0 & 0 & 0 & 0 & 1 & 0 & 0 & 0 & 0 & 0 & 0 & 1 & Ros den \\
\hline \multicolumn{15}{|l|}{ Calymperaceae } \\
\hline Syrrhopodon gaudichaudii Mont.* & $\mathrm{T}$ & 0 & 0 & 1 & 0 & 0 & 0 & 0 & 0 & 0 & 1 & 0 & 0 & Syr gau \\
\hline Syrrhopodon parasiticus (Brid.) Besch. & $\mathrm{T}$ & 0 & 0 & 5 & 1 & 3 & 0 & 0 & 7 & 0 & 2 & 0 & 0 & Syr par \\
\hline \multicolumn{15}{|l|}{ Cryphaeaceae } \\
\hline Schoenobryum concavifolium (Griff.) Gangulee* & $\mathrm{T}$ & 0 & 0 & 3 & 0 & 0 & 0 & 0 & 3 & 0 & 0 & 0 & 0 & Sch conv \\
\hline \multicolumn{15}{|l|}{ Dicranaceae } \\
\hline Campylopus flexuosus (Hedw.) Brid.* & $\mathrm{T}$ & 1 & 0 & 0 & 0 & 0 & 1 & 0 & 0 & 0 & 0 & 0 & 0 & Cam fle \\
\hline Campylopus introflexus (Hedw.) Brid. & $\mathrm{T}$ & 5 & 1 & 0 & 0 & 0 & 6 & 0 & 0 & 0 & 0 & 0 & 0 & Cam int \\
\hline Campylopus occultus Mitt. & $\mathrm{T}$ & 4 & 0 & 0 & 0 & 1 & 4 & 0 & 0 & 0 & 0 & 0 & 1 & Cam occ \\
\hline Campylopus surinamensis Müll. Hal.* & $\mathrm{T}$ & 0 & 0 & 0 & 0 & 1 & 0 & 0 & 0 & 0 & 0 & 0 & 1 & Cam sur \\
\hline \multicolumn{15}{|l|}{ Fabroniaceae } \\
\hline Fabronia ciliaris var. polycarpa (Hook.) W.R. Buck* & W & 0 & 0 & 0 & 0 & 1 & 0 & 0 & 1 & 0 & 0 & 0 & 0 & Fab cil \\
\hline \multicolumn{15}{|l|}{ Fissidentaceae } \\
\hline Fissidens angustifolius Sull.* & $\mathrm{T}$ & 0 & 0 & 0 & 0 & 1 & 0 & 1 & 0 & 0 & 0 & 0 & 0 & Fis ang \\
\hline Fissidens curvatus Hornsch.* & $\mathrm{T}$ & 0 & 0 & 0 & 1 & 0 & 0 & 0 & 0 & 0 & 0 & 1 & 0 & Fis cur \\
\hline Fissidens elegans Brid. & $\mathrm{T}$ & 0 & 0 & 0 & 2 & 2 & 0 & 1 & 0 & 0 & 0 & 3 & 0 & Fis ele \\
\hline Fissidens intromarginatus (Hampe) Mitt.* & $\mathrm{T}$ & 0 & 0 & 0 & 0 & 1 & 0 & 0 & 0 & 0 & 0 & 1 & 0 & Fis int \\
\hline Fissidens serratus Müll. Hal. & $\mathrm{T}$ & 0 & 0 & 1 & 0 & 1 & 0 & 1 & 1 & 0 & 0 & 0 & 0 & Fis ser \\
\hline Fissidens zollingeri Mont.* & $\mathrm{T}$ & 0 & 0 & 0 & 0 & 3 & 0 & 2 & 0 & 0 & 0 & 0 & 1 & Fis zol \\
\hline \multicolumn{15}{|l|}{ Hypnaceae } \\
\hline Chryso-hypnum diminutivum (Hampe) W.R. Buck & W & 0 & 1 & 0 & 0 & 1 & 0 & 0 & 1 & 0 & 1 & 0 & 0 & Chr dim \\
\hline \multicolumn{15}{|l|}{ Hypopterygiaceae } \\
\hline Hypopterygium tamarisci (Sw.) Brid. ex Müll.Hal. & $\mathrm{D}$ & 0 & 0 & 0 & 1 & 1 & 0 & 0 & 1 & 0 & 0 & 1 & 0 & Hyp tam \\
\hline \multicolumn{15}{|l|}{ Meteoriaceae } \\
\hline Floribundaria flaccida (Mitt.) Broth.* & $\mathrm{P}$ & 0 & 0 & 0 & 0 & 1 & 0 & 0 & 1 & 0 & 0 & 0 & 0 & Flo fla \\
\hline Meteorium nigrescens (Sw. ex Hedw.) Dozy\&Molk. & $\mathrm{P}$ & 0 & 0 & 0 & 1 & 4 & 0 & 0 & 5 & 0 & 0 & 0 & 0 & Met nig \\
\hline Neckeraceae & & & & & & & & & & & & & & \\
\hline Neckeropsis undulata (Hedw.) Reichardt & $\mathrm{P}$ & 0 & 0 & 1 & 0 & 3 & 0 & 0 & 4 & 0 & 0 & 0 & 0 & Nec und \\
\hline Orthostichellaceae & & & & & & & & & & & & & & \\
\hline Orthostichella pachygastrella (Müll.Hal.) B.H. Allen \& Magill & $\mathrm{P}$ & 0 & 0 & 1 & 1 & 1 & 0 & 0 & 3 & 0 & 0 & 0 & 0 & Ort pac \\
\hline Orthostichella rigida (Müll. Hal.) B. H. Allen \& Magill* & $\mathrm{P}$ & 0 & 0 & 0 & 1 & 0 & 0 & 0 & 1 & 0 & 0 & 0 & 0 & Ort rig \\
\hline Orthostichella versicolor (Müll.Hal.) B.H. Allen \& W.R. Buck* & $\mathrm{P}$ & 0 & 0 & 0 & 1 & 0 & 0 & 0 & 1 & 0 & 0 & 0 & 0 & Ort ver \\
\hline Orthotrichaceae & & & & & & & & & & & & & & \\
\hline Groutiella apiculata (Hook.) H.A. Crum \& Steere* & M & 0 & 0 & 1 & 0 & 0 & 0 & 0 & 1 & 0 & 0 & 0 & 0 & Gro api \\
\hline Macromitrium richardii Schwägr. & W & 0 & 3 & 7 & 0 & 2 & 0 & 0 & 12 & 0 & 0 & 0 & 0 & Mac ric \\
\hline Macromitrium sharpii CrumexVitt* & W & 0 & 0 & 0 & 0 & 1 & 0 & 0 & 1 & 0 & 0 & 0 & 0 & Mac sha \\
\hline Schloteimia jamesonii (Arn.) Brid. & W & 0 & 0 & 2 & 0 & 2 & 0 & 0 & 4 & 0 & 0 & 0 & 0 & Schl jam \\
\hline Schloteimia rugifolia (Hook.) Schwägr. & W & 0 & 0 & 1 & 0 & 5 & 0 & 0 & 5 & 0 & 1 & 0 & 0 & Schl rug \\
\hline
\end{tabular}


Table 1. Cont.

\begin{tabular}{|c|c|c|c|c|c|c|c|c|c|c|c|c|c|c|}
\hline \multirow[b]{2}{*}{ Taxon } & \multirow{2}{*}{ 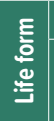 } & \multicolumn{5}{|c|}{ Phytophysiognomies } & \multicolumn{7}{|c|}{ Bryocenological groups } & \multirow[b]{2}{*}{$\begin{array}{c}\text { Abbreviation of the } \\
\text { taxon }\end{array}$} \\
\hline & & 로 & 荇 & 蔹 & 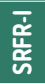 & 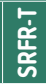 & $\stackrel{\circ}{<}$ & $\mathbb{J}$ & 8 & 욘 & x & ๕ & $\ddot{\mapsto}$ & \\
\hline \multicolumn{15}{|l|}{ Phyllogoniaceae } \\
\hline Phyllogonium viride Brid.* $^{*}$ & $\mathrm{P}$ & 0 & 0 & 3 & 0 & 0 & 0 & 0 & 3 & 0 & 0 & 0 & 0 & Phy vir \\
\hline \multicolumn{15}{|l|}{ Pilotrichaceae } \\
\hline Callicostella martiana (Hornsch.) A. Jaeger* & M & 0 & 0 & 0 & 0 & 1 & 0 & 1 & 0 & 0 & 0 & 0 & 0 & Call mar \\
\hline \multicolumn{15}{|l|}{ Pottiaceae } \\
\hline Tortella humilis (Hedw.) Jenn. & $\mathrm{T}$ & 0 & 1 & 0 & 0 & 3 & 1 & 2 & 0 & 0 & 1 & 0 & 0 & Tor hum \\
\hline \multicolumn{15}{|l|}{ Pterobryaceae } \\
\hline Orthostichopsis tenuis (A. Jaeger) Broth. & $\mathrm{P}$ & 0 & 0 & 3 & 0 & 1 & 0 & 0 & 4 & 0 & 0 & 0 & 0 & Orth tenu \\
\hline Orthostichopsis tijucae (Müll.Hal.) Broth. & $\mathrm{P}$ & 0 & 0 & 4 & 1 & 0 & 0 & 0 & 5 & 0 & 0 & 0 & 0 & Orth tij \\
\hline Pterobryon densum Hornsch.* & $\mathrm{D}$ & 0 & 0 & 1 & 0 & 0 & 0 & 0 & 1 & 0 & 0 & 0 & 0 & Pte den \\
\hline \multicolumn{15}{|l|}{ Pylaisiadelphaceae } \\
\hline Isopterygium subbrevisetum (Hampe) Broth.* & M & 0 & 0 & 1 & 0 & 0 & 0 & 0 & 0 & 0 & 1 & 0 & 0 & Iso sub \\
\hline Isopterygium tenerifolium Mitt.* & M & 0 & 0 & 0 & 2 & 0 & 0 & 0 & 1 & 0 & 1 & 0 & 0 & Iso tenf \\
\hline Isopterygium tenerum (Sw.) Mitt. & M & 1 & 0 & 1 & 9 & 7 & 1 & 0 & 8 & 0 & 5 & 0 & 4 & Iso tene \\
\hline \multicolumn{15}{|l|}{ Rachitheciaeceae } \\
\hline Uleastrum palmicola (Müll.Hal.) R.H.Zander* & $\mathrm{T}$ & 0 & 0 & 0 & 0 & 1 & 0 & 0 & 1 & 0 & 0 & 0 & 0 & Ule pal \\
\hline \multicolumn{15}{|l|}{ Sematophyllaceae } \\
\hline Brittonodoxa subpinnata (Brid.) W.R. Buck, P.E.A.S.Câmara \& Carv.-Silva & $\mathrm{M}$ & 0 & 6 & 8 & 4 & 15 & 1 & 0 & 27 & 0 & 3 & 1 & 1 & Bri sub \\
\hline \multicolumn{15}{|l|}{ Sphagnaceae } \\
\hline Sphagnum palustre L. & $\mathrm{T}$ & 3 & 0 & 1 & 0 & 0 & 0 & 0 & 0 & 0 & 0 & 0 & 4 & Sph pal \\
\hline Sphagnum recurvum L.P.Beauv.* & $\mathrm{T}$ & 0 & 0 & 1 & 0 & 0 & 0 & 0 & 0 & 0 & 0 & 0 & 1 & Sph rec \\
\hline \multicolumn{15}{|l|}{ MARCHANTIOPHYTA } \\
\hline Aneuraceae & & & & & & & & & & & & & & \\
\hline Riccardia regnellii (Angstr.) G.K.Hell & Th & 0 & 0 & 0 & 1 & 3 & 0 & 2 & 0 & 0 & 1 & 0 & 1 & Ric reg \\
\hline Cephaloziellaceae & & & & & & & & & & & & & & \\
\hline Cylindrocolea planifolia (Steph.) R.M. Schust.* & W & 2 & 0 & 0 & 0 & 0 & 2 & 0 & 0 & 0 & 0 & 0 & 0 & Cyl pla \\
\hline Cylindrocolea rhizantha (Mont.) R.M. Schust.* & W & 2 & 0 & 0 & 0 & 0 & 2 & 0 & 0 & 0 & 0 & 0 & 0 & Cyl rhi \\
\hline Frullaniaceae & & & & & & & & & & & & & & \\
\hline Frullania brasiliensis Raddi* & $\mathrm{P}$ & 0 & 0 & 0 & 0 & 1 & 0 & 0 & 1 & 0 & 0 & 0 & 0 & Fru bra \\
\hline Frullania caulisequa (Nees) Nees & M & 0 & 0 & 0 & 1 & 3 & 0 & 0 & 4 & 0 & 0 & 0 & 0 & Fru cau \\
\hline Frullania ericoides (Nees) Mont. & M & 0 & 0 & 0 & 2 & 2 & 0 & 0 & 4 & 0 & 0 & 0 & 0 & Fru eri \\
\hline Frullania griffithsiana Gottsche* & M & 0 & 0 & 1 & 0 & 0 & 0 & 0 & 1 & 0 & 0 & 0 & 0 & Fru gri \\
\hline Frullania riojaneirensis (Raddi) Spruce* & M & 0 & 0 & 2 & 0 & 0 & 0 & 0 & 1 & 0 & 1 & 0 & 0 & Fru rio \\
\hline Lejeuneaceae & & & & & & & & & & & & & & \\
\hline Acanthocoleus trigonus (Nees \& Mont.) Gradst. & M & 0 & 0 & 0 & 5 & 7 & 0 & 0 & 12 & 0 & 0 & 0 & 0 & Aca tri \\
\hline Bryopteris filicina (Sw.) Nees* & $\mathrm{P}$ & 0 & 0 & 0 & 0 & 2 & 0 & 0 & 2 & 0 & 0 & 0 & 0 & Bryo fil \\
\hline Cheilolejeunea rigidula (Nees ex Mont.) R.M. Schust.* & M & 0 & 0 & 1 & 0 & 0 & 0 & 0 & 1 & 0 & 0 & 0 & 0 & Che rig \\
\hline Cheilolejeunea unciloba (Lindenb.) Malombe* & M & 0 & 0 & 1 & 0 & 0 & 0 & 0 & 1 & 0 & 0 & 0 & 0 & Che unc \\
\hline Cheilolejeunea xanthocarpa (Lehm. \& Lindenb.) Malombe* & M & 0 & 0 & 1 & 0 & 0 & 0 & 0 & 1 & 0 & 0 & 0 & 0 & Che xan \\
\hline Cololejeunea clavatopapillata Steph.* & M & 0 & 0 & 1 & 0 & 0 & 0 & 0 & 0 & 0 & 1 & 0 & 0 & Col cla \\
\hline Cololejeunea minutilobula Herzog* & M & 0 & 0 & 0 & 3 & 0 & 0 & 0 & 0 & 3 & 0 & 0 & 0 & Col min \\
\hline Drepanolejeunea anoplantha (Spruce) Steph.* & M & 0 & 0 & 0 & 0 & 1 & 0 & 0 & 1 & 0 & 0 & 0 & 0 & Dre ano \\
\hline Drepanolejeunea fragilis Bischl.* & M & 0 & 0 & 1 & 0 & 0 & 0 & 0 & 0 & 0 & 1 & 0 & 0 & Dre fra \\
\hline Lejeunea adpressa Nees & M & 0 & 0 & 0 & 1 & 1 & 0 & 0 & 2 & 0 & 0 & 0 & 0 & Lej adp \\
\hline Lejeunea aphanes Spruce* & M & 0 & 1 & 0 & 0 & 0 & 0 & 0 & 1 & 0 & 0 & 0 & 0 & Lej aph \\
\hline Lejeunea bermudiana (A.Evans) R.M.Schust. & M & 0 & 0 & 0 & 1 & 2 & 0 & 0 & 3 & 0 & 0 & 0 & 0 & Lej ber \\
\hline Lejeunea cancellata Nees \& Mont.* & M & 0 & 0 & 0 & 0 & 2 & 0 & 0 & 2 & 0 & 0 & 0 & 0 & Lej can \\
\hline Lejeunea flava (Sw.) Nees & M & 0 & 0 & 3 & 5 & 3 & 0 & 0 & 9 & 0 & 2 & 0 & 0 & Lej fla \\
\hline Lejeunea laeta (Lehm. \& Lindenb.) Gottsche* & M & 0 & 0 & 3 & 0 & 0 & 0 & 0 & 3 & 0 & 0 & 0 & 0 & Lej leta \\
\hline Lejeunea laetevirens Nees \& Mont. & M & 0 & 0 & 5 & 1 & 3 & 0 & 0 & 9 & 0 & 0 & 0 & 0 & Lej lae \\
\hline Lejeunea monimiae (Steph.) Steph.* & M & 0 & 0 & 0 & 0 & 2 & 0 & 0 & 1 & 0 & 1 & 0 & 0 & Lej mon \\
\hline Lejeunea phyllobola Nees \& Mont. & M & 0 & 0 & 3 & 3 & 2 & 0 & 0 & 8 & 0 & 0 & 0 & 0 & Lej phy \\
\hline Lejeunea setiloba Spruce* & M & 0 & 2 & 0 & 0 & 0 & 1 & 0 & 1 & 0 & 0 & 0 & 0 & Lej set \\
\hline Lopholejeunea nigricans (Lindenb.) Schiffn.* & M & 0 & 0 & 0 & 1 & 0 & 0 & 0 & 0 & 0 & 1 & 0 & 0 & Lop nig \\
\hline
\end{tabular}


Table 1. Cont.

\begin{tabular}{|c|c|c|c|c|c|c|c|c|c|c|c|c|c|c|}
\hline \multirow[b]{2}{*}{ Taxon } & \multirow{2}{*}{ 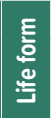 } & \multicolumn{5}{|c|}{ Phytophysiognomies } & \multicolumn{7}{|c|}{ Bryocenological groups } & \multirow{2}{*}{$\begin{array}{c}\text { Abbreviation of the } \\
\text { taxon }\end{array}$} \\
\hline & & 로 & 咅 & 旁 & 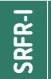 & 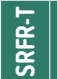 & $\stackrel{\propto}{<}$ & 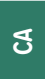 & 8 & 욥 & x & 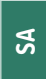 & 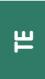 & \\
\hline Microlejeunea epiphylla Bischl. & M & 0 & 0 & 0 & 2 & 1 & 0 & 0 & 1 & 2 & 0 & 0 & 0 & Mic epi \\
\hline Microlejeunea subulistipa Steph.* & M & 0 & 0 & 1 & 0 & 0 & 0 & 0 & 1 & 0 & 0 & 0 & 0 & Mic sub \\
\hline Schiffneriolejeunea polycarpa (Nees) Gradst.* & M & 0 & 0 & 0 & 0 & 2 & 0 & 0 & 2 & 0 & 0 & 0 & 0 & Schi pol \\
\hline \multicolumn{15}{|l|}{ Lepidoziaceae } \\
\hline Telaranea nematodes (Gottscheex Austin) M.A.Howe* & W & 0 & 0 & 1 & 0 & 0 & 0 & 0 & 1 & 0 & 0 & 0 & 0 & Tel nem \\
\hline \multicolumn{15}{|l|}{ Lophocoleaceae } \\
\hline Lophocolea bidentata (L.) Dumort.* & M & 0 & 0 & 0 & 0 & 1 & 0 & 1 & 0 & 0 & 0 & 0 & 0 & Loph bid \\
\hline \multicolumn{15}{|l|}{ Metzgeriaceae } \\
\hline Metzgeria aurantiaca Steph.* & Th & 0 & 0 & 0 & 0 & 1 & 0 & 0 & 1 & 0 & 0 & 0 & 0 & Metz aur \\
\hline Metzgeria brasiliensis Schiffn. & Th & 0 & 1 & 0 & 1 & 1 & 0 & 0 & 2 & 0 & 1 & 0 & 0 & Metz bra \\
\hline Metzgeria conjugata Lindb.* & Th & 0 & 0 & 0 & 0 & 1 & 0 & 0 & 1 & 0 & 0 & 0 & 0 & Metz conj \\
\hline Metzgeria convoluta Steph. & Th & 0 & 0 & 1 & 0 & 3 & 0 & 0 & 4 & 0 & 0 & 0 & 0 & Metz conv \\
\hline Metzgeria furcata (L.) Dumort. & Th & 0 & 0 & 0 & 4 & 1 & 0 & 0 & 5 & 0 & 0 & 0 & 0 & Metz fur \\
\hline Metzgeria myriopoda Lindb. & Th & 0 & 0 & 1 & 0 & 1 & 0 & 0 & 2 & 0 & 0 & 0 & 0 & Metz myr \\
\hline Metzgeria rufula Spruce* & Th & 0 & 0 & 0 & 0 & 2 & 0 & 0 & 2 & 0 & 0 & 0 & 0 & Metz ruf \\
\hline \multicolumn{15}{|l|}{ Pallaviciniaceae } \\
\hline Symphyogyna brasiliensis (Nees) Nees \& Mont.* & Th & 2 & 0 & 0 & 0 & 0 & 2 & 0 & 0 & 0 & 0 & 0 & 0 & Sym bra \\
\hline \multicolumn{15}{|l|}{ Plagiochilaceae } \\
\hline Plagiochila corrugata (Nees) Nees \& Mont. & $\mathrm{P}$ & 0 & 0 & 2 & 1 & 9 & 0 & 0 & 11 & 0 & 1 & 0 & 0 & Pla cor \\
\hline Plagiochila crispabilis Lindenb.* & $\mathrm{P}$ & 0 & 0 & 0 & 0 & 2 & 0 & 0 & 2 & 0 & 0 & 0 & 0 & Pla cri \\
\hline Plagiochila disticha (Lehm. \& Lindenb.) Lindenb.* & $\mathrm{P}$ & 0 & 0 & 0 & 0 & 1 & 0 & 0 & 1 & 0 & 0 & 0 & 0 & Pla dis \\
\hline Plagiochila martiana (Nees) Lindenb. & $\mathrm{P}$ & 0 & 0 & 0 & 2 & 1 & 0 & 0 & 3 & 0 & 0 & 0 & 0 & Pla mar \\
\hline Plagiochila montagnei Nees* & $\mathrm{P}$ & 0 & 0 & 0 & 0 & 1 & 0 & 0 & 1 & 0 & 0 & 0 & 0 & Pla mon \\
\hline Plagiochila raddiana Lindenb.* & $\mathrm{P}$ & 0 & 0 & 0 & 1 & 0 & 0 & 0 & 0 & 0 & 1 & 0 & 0 & Pla rad \\
\hline \multicolumn{15}{|l|}{ Radulaceae } \\
\hline Radula javanica Gottsche* & M & 0 & 0 & 3 & 0 & 0 & 0 & 0 & 3 & 0 & 0 & 0 & 0 & Rad jav \\
\hline Radula nudicaulis Steph.* & M & 0 & 0 & 1 & 0 & 0 & 0 & 0 & 1 & 0 & 0 & 0 & 0 & Rad nud \\
\hline Radula tectiloba Steph.* & M & 0 & 0 & 3 & 0 & 0 & 0 & 0 & 3 & 0 & 0 & 0 & 0 & Rad tec \\
\hline
\end{tabular}

Approximately $50 \%$ of the species in HL (five) and in SWRF (20) were exclusive to those sites. The smallest percentage of exclusivity was seen in SRFD (four species), approximately $36 \%$.

Considering the species identified in SRFR, there were differences in the richness and floristic composition between SRFR-T and SRFR-I. Among the 67 species encountered in SRFR, 36 were found only in SRFR-T and eight only in SRFR-I; 23 species were shared by both areas. The species encountered only in SRFR-I were Fissidens curvatus, Orthostichella rigida, Orthostichella versicolor, Orthostichopsis tijucae, Isopterygium tenerifolium, Cololejeunea minutilobula, Lopholejeunea nigricans, and Plagiochila raddiana.

\section{Floristic similarities between the different phytophysiognomies}

There were pronounced floristic differences between the phytophysiognomies, as evidenced by the low Jaccard values $(<0.35)$. The greatest similarities were between SRFR-I and SRFR-T (0.34), which are actually two portions of the same phytophysiognomy. HL was the most floristically distinct phytophysiognomy $(<0.05)$.
The biplot diagram of the CA (axes 1 and $2=58.9 \%$ of the variance) corroborated the data of the grouping analysis, evidencing the greater similarity between SRFR-I and SRFR-T, and the generally elevated floristic distinction among all of the phytophysiognomies examined. That grouping analysis diagram indicates which species predominate in each of the different phytophysiognomies, with special attention to the high number of exclusive species in SRFR-I (Fig. 3). The separation of HL from the other phytophysiognomies can be seen along axis I; axis 2 evidences the floristic differences among SRFD, SRFR and SWRF.

\section{Bryocenological groups}

The species collected in the different phytophysiognomies of the PEVA were colonizing different substrates. The most expressive bryocenological groups in terms of their numbers of species were corticolous (68 species), epixylic (22), arenicolous (13), and terricolous (11); chasmophytes were represented by only eight species, with six saxicolous species, but only two epiphyllous taxa (Tab. 2).

In terms of their substrate preferences, 76 species demonstrated preference for only a single substrate type, 
predominating the corticolous bryocenological group (51 species). The other groups demonstrated less than 10 species within a given preference, being arenicolous and epixylic (seven each), terricolous (five), chasmophytes (three), saxicolous (two), and epiphyllous (one).

The species that did not demonstrate preferences for only a single substrate, also did not occupy a wide variety of surfaces (Tab. 2), that is, there was a low similarity of species in terms of the substrates sampled $(<0.2)$. The largest similarity indices were observed between the sandy substrate and soils (0.2), living and decomposing tree trunks (0.17), and between rock surfaces and soils (0.13). Leaves demonstrated the greatest floristic differentiation as a substrate $(<0.01)$.

The CA diagram evidenced the high diversity of species that occur exclusively as epiphytes (Fig. 4), with the first two axes representing $47.5 \%$ of the total variance. The separation of the corticolous and epixylic groups of species from the others along axis 1 can easily be seen. Axis 2 demonstrates the distinction between species that colonize artificial substrates ("chasmophyte" (upper left quadrant)) and sandy sediments ("arenicolous" (lower left quadrant)). Those analyses were made excluding the epiphyllous bryocenological group because of their low sharing of species. Species richness per bryocenological group in each phytophysiognomy (Tab. 3) evidenced a greater variety of substrates colonized in SRFR-T and SRFR-I. Only three different substrates were colonized in SRFD, two in SWRF, and only one in HL.

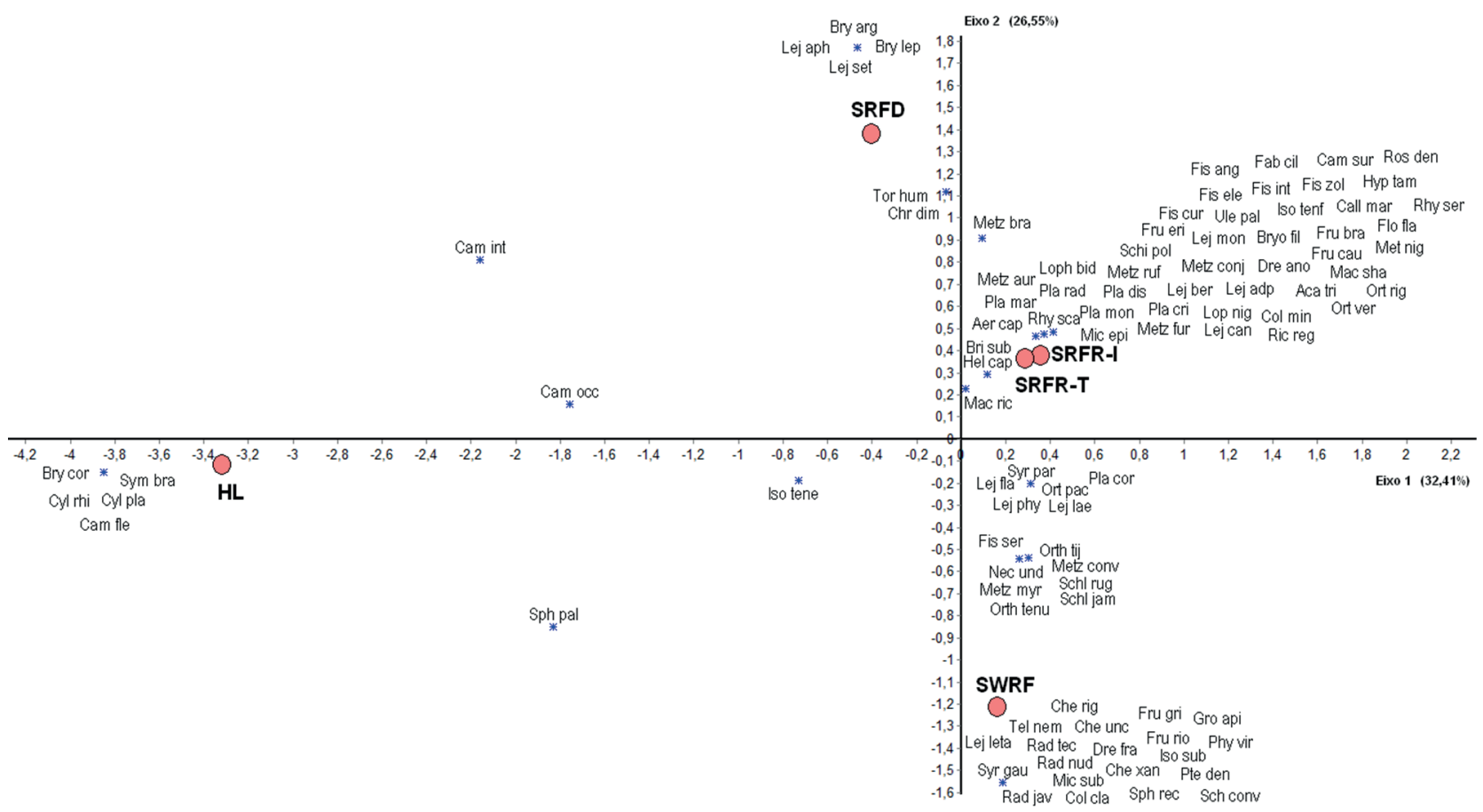

Figure 3. Biplot diagram of the Correspondence Analysis (CA) with the phytophysiognomies distributed according to their species compositions of bryophytes in the Itapeva State Park, Torres, Rio Grande do Sul State, Brazil. HL = humid lowlands. SRFD= sandy Restinga forest growing on dunes. SWRF= swampy Restinga forest. SRFR-I= sandy Restinga forest growing on rock outcrops (interior). SRFR-T = sandy Restinga forest growing on rock outcrops (trail). Abbreviation of the taxon according to Table 1.

Table 2. Comparisons between bryophyte richness in the seven bryocenological groups encountered in the Itapeva State Park, Torres, Rio Grande do Sul State, Brazil. Bold type = number of species per bryocenological group; normal type = number of species in common among the groups; italics $=$ Jaccard similarity index. $\mathrm{AR}=$ arenicolous. $\mathrm{CA}=$ chasmophyte. $\mathrm{CO}=$ corticolous. $\mathrm{EP}=$ epiphyllous . $\mathrm{EX}=$ epixylic. $\mathrm{SA}=$ saxicolous. $\mathrm{TE}=$ terricolous.

\begin{tabular}{|c|c|c|c|c|c|c|c|}
\hline & AR & CA & CO & EP & EX & SA & TE \\
\hline AR & $\mathbf{1 3}$ & 1 & 4 & 0 & 4 & 2 & 2 \\
\hline CA & 0.05 & $\mathbf{8}$ & 1 & 0 & 2 & 1 & 2 \\
\hline CO & 0.05 & 0.01 & $\mathbf{6 8}$ & 1 & 13 & 3 \\
\hline EP & 0.00 & 0.00 & 0.01 & $\mathbf{2}$ & 0 & 0 & 0 \\
\hline EX & 0.129 & 0.07 & 0.17 & 0.00 & $\mathbf{2 2}$ & 2 & 4 \\
\hline SA & 0.12 & 0.08 & 0.04 & 0.00 & 0.08 & $\mathbf{6}$ & 2 \\
\hline TE & 0.20 & 0.12 & 0.04 & 0.00 & 0.14 & 0.13 & $\mathbf{1 1}$ \\
\hline
\end{tabular}




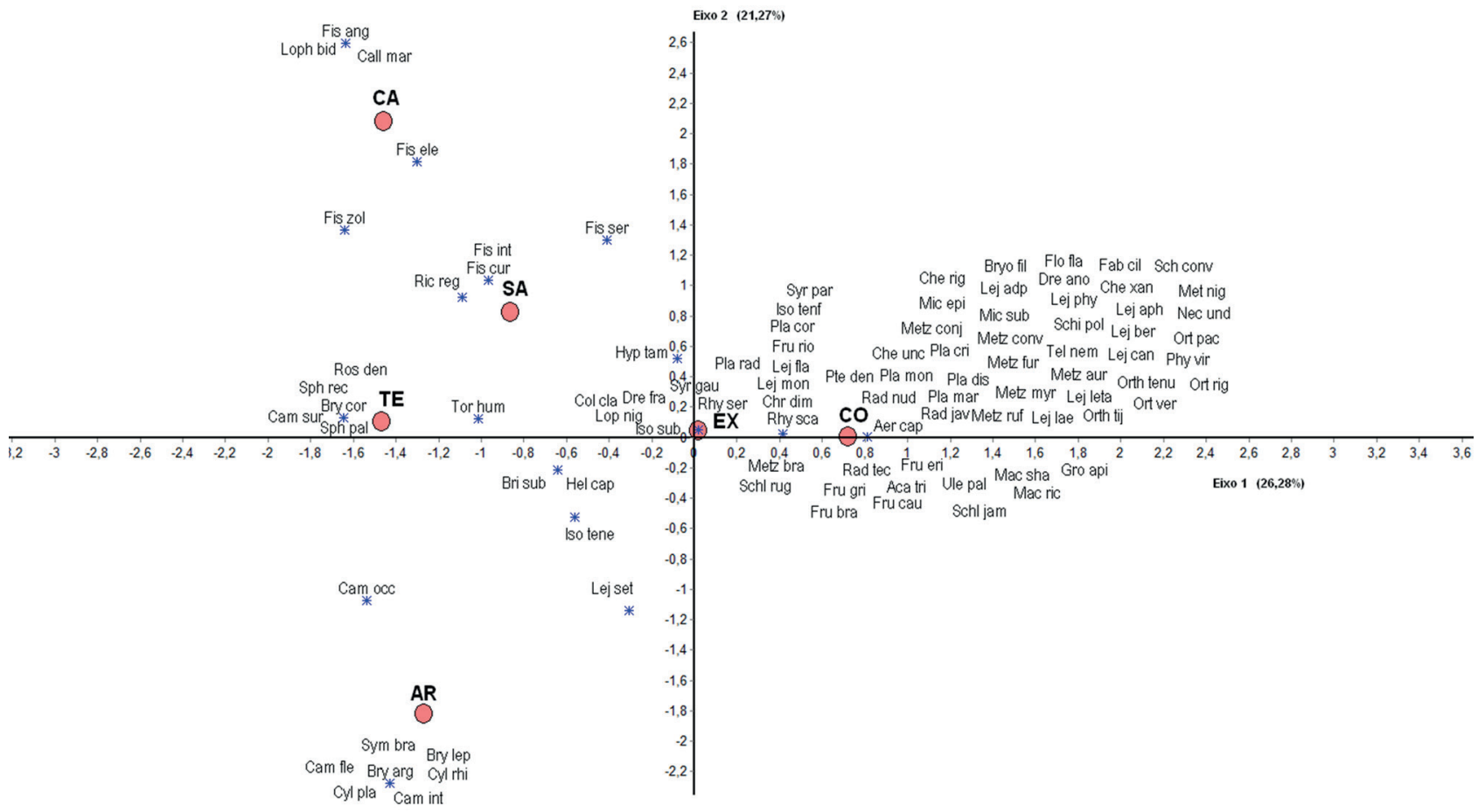

Figure 4. Biplot diagram of the Correspondence Analysis (CA) of the data on species incidence related to their substrate preferences (except epiphyllous species) in the Itapeva State Park, Torres, Rio Grande do Sul State, Brazil. AR= arenicolous. CA= chasmophyte. $\mathrm{CO}=$ corticolous. $\mathrm{EX}=$ epixylic. $\mathrm{SA}=$ saxicolous. $\mathrm{TE}=$ terricolous. Abbreviation of the taxon according to Table 1.

Table 3. Species richness of bryophytes per bryocenological group in each phytophysiognomy in the Itapeva State Park, Torres, Rio Grande do Sul State, Brazil. HL = humid lowlands. SRFD= sandy Restinga forest growing on dunes. SWRF= swampy Restinga forest. SRFR-I= sandy Restinga forest growing on rock outcrops (interior). SRFR-T= sandy Restinga forest growing on rock outcrops (trail). $\mathrm{AR}=$ arenicolous. $\mathrm{CA}=$ chasmophyte $\mathrm{CO}=$ corticolous. $\mathrm{EP}=$ epiphyllous. $\mathrm{EX}=$ epixylic. $\mathrm{SA}=$ saxicolous. $\mathrm{TE}=$ terricolous.

\begin{tabular}{|c|c|c|c|c|c|}
\hline & HL & SRFD & SRFR-I & SRFR-T & SWRF \\
\hline AR & 7 & 7 & 0 & 0 & 0 \\
\hline CA & 0 & 0 & 0 & 45 & 0 \\
\hline EP & 0 & 7 & 21 & 0 & 33 \\
\hline EX & 0 & 0 & 2 & 10 & 0 \\
\hline SA & 0 & 1 & 9 & 3 & 7 \\
\hline TE & 0 & 0 & 0 & 8 & 0 \\
\hline
\end{tabular}

\section{Discussion}

\section{(i) Species richness in the phytophysiognomies}

The floristic richness of the bryoflora encountered in the PEVA corresponded to $17 \%$ of that known for Rio Grande do Sul State and $6 \%$ of all of Brazil (Costa \& Peralta 2015). Fourteen species encountered in the area were new occurrences for the state (Bordin et al. 2020; TS Dewes unpubl.res.). The Restinga forests (SRFR and SWRF) were the richest PEVA phytophysiognomies. Both areas were forest types that demonstrate high environmental heterogeneity and many micro-habitats appropriate for bryophyte colonization (Pócs 1982; Waechter 1985; Frahm 2003; Duarte \& Bencke 2006), which was corroborated by the greater colonization of substrates there and greater varieties of life forms.

Záchia \& Waechter (2011) undertook an ecological study of the herbaceous/shrub phanerogam communities in Restinga vegetation in the southern region of Rio Grande do Sul State, and encountered the greatest species richness in sandy soil forests (much like those of the SRFR), as compared to seasonally flooded forests (such as SWRF). Santos-Junior et al. (2015), on the other hand, reported low diversity estimator values in a swampy forest along the northern coast of that same state as compared to Dense Ombrophilous forests in other states. That tendency of swampy forests to have less species richness than well-drained soils in other forest types has been corroborated by other authors (Sztutman \& Rodrigues 2002; Silva et al. 2007; Martins et al. 2013). The 
species richness of swampy forests tends to increase with increased proximity to Dense Ombrophilous forests and diminishing soil water saturation (Mancino et al. 2015).

The SRFD and HL phytophysiognomies have herbaceous/ shrub and herbaceous vegetation covers respectively (Waechter 1985; Duarte \& Bencke 2006), characterizing more homogeneous environments than those found in forests. As those two phytophysiognomies are located in sandy coastal dune areas, they are more exposed to stressful abiotic conditions (such as salinity, wind, high soil mobility, scarcity of nutrients, water restrictions, high solar radiation, and high temperatures) (Scott 1982), which will result in lower local indices of humidity and much higher indices of solar radiation that can interfere with bryophyte development (Mägdefrau 1982).

Although composing two parts of the same phytophysiognomy, the SRFR-I and SRFR-T areas demonstrated significantly different species richness (Fig. 2A). Although microclimatic variations were not quantified in this study, edge environments (such as along trails) are known to have distinct conditions of luminosity, humidity, and temperature in relation to forest interiors - important factors for tropical bryophyte species distributions (Richards 1984; Silva \& Pôrto 2009; 2010; 2013; Souza et al. 2020).

\section{(ii) Species compositions, life forms, and floristic similarities of the phytophysiognomies}

The low observed similarity between the different phytophysiognomies in the present study is directly related to the floristic singularities of the different areas. The families with the greatest number of bryophyte species in SRFR (Lejeuneaceae, Metzgeriaceae, Fissidentaceae, and Plagiochilaceae) are representative tropical forest families and among the richest in terms of their numbers of species in Brazil (Costa \& Peralta 2015). Lejeuneaceae also stood out in terms of its high numbers of species in SWRF and SRFD. Liverworts, especially members of that family, are among the predominant bryophytes found in upland tropical forests (Gradstein et al. 2001). The family Dicranaceae had the largest number of species in HL (those species belong to the genus Campylopus, and also occur in SRFR-T and SRFD). According to Frahm (1991), most species of that family are characterized by an intolerance of shading and a preference for open environments and substrates having $\mathrm{pH}<6-$ environmental conditions corresponding to those of sandy coastal dunes (Scott 1982) of those phytophysiognomies.

The species shared by a number of phytophysiognomies in the present study demonstrate wide geographic distributions, varying between Neotropical (Helicodontium capillare, Isopterygium tenerum, and Macromitrium richardii) and Pantropical (Brittonodoxa subpinnata) (Costa et al. 2011). All of those species occur in the Brazilian Atlantic Forest, in the Amazon region, and in dryer phytogeographic domains with shrub-arboreal vegetation, such as Caatinga, Cerrado, and Pampa (except for M. richardii) (Costa 2010).
Additionally, $H$. capillare and $M$. richardii are typical sun species, while B. subpinnata and I. tenerum are generalists (Oliveira et al. 2011; Santos et al. 2011; Batista et al. 2018). Those characteristics demonstrate their tolerance to diverse microclimatic conditions and would explain their presence in the distinct phytophysiognomies found in the PEVA.

Most of the species (74) were exclusive to just a single phytophysiognomy. That exclusivity was most expressive in SRFR, probably due to its environmental heterogeneity and large number of microenvironments available for colonization, with different moisture and light conditions, different types of living and decaying logs, soils, and other substrates. The epiphyllous species Cololejeunea minutilobula and Microlejeunea epiphylla and saxicolous species Fissidens curvatus and Fissidens intromarginatus, for example, occurred exclusively in that phytophysiognomy. The SWRF had 20 exclusive species, including Pterobryon densum, Sphagnum recurvum, Drepanolejeunea fragilis, Lejeunea laeta, and Microlejeunea subulistipa.

Some species occurred exclusively in HL (five species) and SRFD (four). Those taxa are not restricted to the Atlantic Forest, but occur in at least one other phytogeographic domain in Brazil, such as the Amazon, Caatinga, Cerrado, or Pantanal (Costa 2010). The geographic distributions of those species vary from Neotropical (Cylindrocolea planifolia, C. rhizantha, Symphyogyna brasiliensis, Lejeunea aphanes, and L. setiloba) (Gradstein \& Costa 2003) to global (Bryum argenteum, B. coronatum, and Campylopus flexuosus) (Frahm 1991; Costa et al. 2011). Bryum atenense is distributed throughout all of the phytogeographic domains in Brazil, except the Amazon and Pampa, and its occurrence was recently amplified to southern Brazil (Bordin et al. 2020).

Different life forms have different tolerances to different microclimatic conditions, principally light and humidity, being considered: tolerant (turf), intermediate (mats, thalloid and weft), or vulnerable (dendroid and pendant) (Gimingham \& Birse 1957; Mägdefrau 1982). Most species found in SRFR-I, SRFR-T, and SWRF demonstrate intermediate tolerances, followed by less-numerous vulnerable and tolerant life forms. The intermediate and tolerant life forms prevailed in SRFD and HL respectively.

The differences in richness and species compositions between the two SRFR areas, as well as the presence of various life forms and degrees of tolerance to luminosity in SRFR-T, corroborate the observation that bryophyte distributions are more related to environmental heterogeneity and the availability of diverse microclimates than to edge distances (Silva \& Pôrto 2009; 2010; 2013).

The occurrence of species belonging to the genera Macromitrium and Frullania in SRFR-T and SWRF suggests that those environments are exposed to higher levels of solar radiation and greater degrees of desiccation. The life forms composing those genera (weft and mat) store water efficiently and are characteristic of habitats experiencing occasional desiccation (Frahm 2003). The 
cell ornamentations found on Macromitrium spp. (cuticular projections known as papilla or mammilla) and the large hyaline cells with thin walls at the base of their leaves, can facilitate water absorption (as well as accelerate its loss) (Proctor 1979; Goffinet et al. 2009; Glime 2017b). The lobes found on the leaves of species of the genus Frullania can absorb and store atmospheric moisture (Gradstein \& Pócs 1989). Only eight species in the SRFR-I were tolerant life forms. The thalloid plants Riccardia regnellii, Metzgeria brasiliensis, and Metzgeria furcata protect one of their sides at the expense of the other, although cuticular substances reduce water losses on the exposed side. Compact growth forms, such as short turf (Syrrhopodon parasiticus, Fissidens curvatus, and Fissidens elegans) are less subject to water losses than open growth forms (Glime 2017b).

The presence and occurrence of species of Meteoriaceae, Neckeraceae, Metzgeria, and Plagiochila in SRFR-T, on the other hand, indicate a more humid environment. The life forms of those species are generally classified as dendroid, thalloid, and pendant, and their gametophytes can be held above the substrate to capture water and light (Gimingham \& Birse 1957; Frahm 2003). Plagiochilaceae and Metzgeriaceae are typical of humid forest areas with highquality environments, that is, high humidity, low luminosity, and low levels of anthropic disturbance (Gradstein et al. 2001; Gradstein \& Costa 2003). Most species of Plagiochila encountered in that section of the SRFR phytophysiognomy are typical shade species and are not tolerant of strong illumination conditions, while some Metzgeria species are generalists (Silva \& Pôrto 2009; 2010; Alvarenga et al. 2010; Oliveira et al. 2011; Santos et al. 2011).

Distinct from the species heterogeneity observed in forest phytophysiognomies, the species compositions in HL and SRFD evidenced the homogeneity of those environments. Scott (1982) described bryophytes with erect growth as tufts (such as Bryum and Campylopus), which are typical of pioneer mosses that predominate under high solar radiation conditions and low humidity - conditions found in the two sandy coastal dune areas of the PEVA. In addition to the compact growth forms less subject to water loss, they can also have morphological characters such as cuticular projections and hyaline cells, as well as lobes for water absorption and retention (Proctor 1979; Gradstein \& Pócs 1989; Goffinet et al. 2009; Glime 2017b). In terms of the life forms and their respective degrees of tolerance to desiccation, most of the species in SRFD were intermediate (64\%), and the rest tolerant (36\%); no species vulnerable to desiccation were encountered. Similarly, tolerant species predominated (56\%) in the HL phytophysiognomy, but with expressive percentages of intermediate species (44\%). The same abiotic factors that restrict the numbers of species growing in hostile environments (such as salinity, wind, high soil mobility, lack of nutrients, water stress, and high solar radiation levels and temperatures) (Mägdefrau 1982; Scott 1982), select for species adapted to those conditions.

\section{(iii) The influence of substrate on species richness and floristic composition}

The occurrence of bryocenological groups will depend on the physical and chemical characteristics of the substrate, as well as the availability and quantities of light, water, and nutrients (Pócs 1982). The corticolous group was most expressive in terms of the number of species (68), followed by epixylics (22). Those results are similar to reports by Gradstein et al. (2001) and Frahm (2003), who described large numbers of epiphytic bryophytes in humid tropical forests - reflecting the variety of temperature, humidity, and luminosity conditions favorable to the colonization of tree trunks by various taxa.

The bryocenological groups encountered in the PEVA demonstrated low similarity, which was directly related to the preference of most species (78\%) for a single type of substrate. The corticolous group was the most expressive in terms of species exclusive to that substrate (51). Diverse and divergent characteristics are observed in those species. Some have mechanisms that aid in water retention, such as small isodiametric leaf cells that curl when dry and expand when wet (such as Groutiella apiculata, Macromitrium richardii, Macromitrium sharpii, Schloteimia jamesonii and Uleastrum palmicola), or have lobes and teeth that serve that same purpose (such as Plagiochila spp.) (Glime 2017c). Pendant and dendroid species (such as Aerolindigia capillacea, Floribundaria flaccida, Meteorium nigrescens, Neckeropsis undulata, Orthostichella spp., Phyllogonium viride, Orthostichopsis spp. and Pterobryon densum), on the other hand, have open life forms and are more subject to water losses (Glime 2017b). Frahm (2003), Germano \& Pôrto (2006), and Batista et al. (2018) observed that the high levels of preference of bryophyte species for a single substrate type in tropical forests reflects their low levels of humidity, as well as filtering for characteristics adapted to each microclimate. The high richness and exclusivity of corticolous species significantly contributed to the richness and floristic composition of the SRFR-T, SRFR-I, and SWRF phytophysiognomies.

The greatest similarity of bryocenological groups was observed among arenicolous and terricolous species, which were predominantly encountered in HL, SRFR-T, and SRFD. Those similarities probably reflect their morphological adaptations for colonizing hostile environments such as sandy soils exposed to conditions of salinity, substrate mobility, low nutrient contents, water stress, and high solar radiation and temperatures (Scott 1982). Among the arenicolous and terricolous species present in those three phytophysiognomies was Campylopus spp., which does not tolerate shade conditions and prefers open environments with acidic substrates (Frahm 1991). Two Sphagnum species were encountered growing on the very humid soil in HL and SWRF, the type of environment that most species of that genus require (Clymo \& Hayward 1982). The soil condition 
in SWRF, together with the absence of rocks and artificial substrates, restricts bryophyte colonization to living or decaying trunks.

Eight different species were encountered growing on soil substrates in SRFR-T. Species of the genera Campylopus, Rosulabryum densifolium, and Fissidens zollingeri demonstrate morphological adaptations such as lamellae, involute margins, hyaline cells, marginal teeth, and elongated leaf border cells that aid in absorbing and storing water (Proctor 1979; Glime 2017d). None of the species encountered in SRFR-I were growing on sand or soil substrates, which was expected, as the floors of humid tropical forests are invariably covered by fallen leaves (Richards 1984). Gradstein \& Pócs (1989) reported that terrestrial species are normally encountered on exposed and/or disturbed soils (substrates not available in SRFR-I) as well as on artificial structures (substrates found in SRFR-T, with eight species). Those eight chasmophyte species, together with the arenicolous and terricolous taxa (eight) partially explain the observed differences in richness and species compositions between SRFR-T and SRFR-I.

The availability of appropriate substrates also influenced the species richness and floristic composition in HL and SRFD. While HL had only seven arenicolous species and two terricolous species, SRFD had seven arenicolous, seven corticolous, and one epixylic species. The vascular plants encountered between the frontal dunes and the mobile interior dunes were strictly herbaceous (Waechter 1985; Duarte \& Bencke 2006), excluding the possibility of corticolous bryophyte colonization in HL. Additionally, the exposed environmental conditions in the sandy coastal dune systems of the HL and SRFD (Scott 1982) severely limited bryophyte colonization and reduced species compositions in those sites. Similarly, Gradstein et al. (2001) reported that species of the genus Cylindrocolea (the same encountered in the present study exclusively in $\mathrm{HL}$ ) are indicators of dry soils in dry forests and savannas. The hyaline portions of the leaves of Bryum (observed in HL and SRFD) represent mechanisms against desiccation and mechanical damage (Scott 1982). The various aspects related to the availability and peculiarities of the substrates reported in the present study suggest their influence on the unique bryofloristic natures of each of the phytophysiognomies examined. Bates (2009) noted that many bryophytes are faithful indicators of specific substrate characteristics, principally their chemical properties, longevity, and water retention capacities.

\section{(iv) The importance of the PEVA for bryophyte conservation}

Protected areas of Brazil are still greatly in need of biodiversity studies (Oliveira et al. 2017). Recent discoveries in Rio Grande do Sul State (Bordin \& Yano 2009a; b; Heidtmann et al. 2013; Weber et al. 2015; Aires et al. 2020; Bordin et al. 2020) evidenced the need for more floristic inventories and evaluations of the conservation statuses of bryophytes throughout the country (Frahm 2003).

Although largely neglected when studies of conservation biology first began, bryophytes have since become increasingly interesting subjects of study in relation to their important ecological roles in water balance, erosion control, nitrogen cycling, and the creation of habitat for other organisms (Vanderpoorten \& Goffinet 2009). Additionally, their potential as indicators of air quality, climatic and soil conditions, the presence of heavy metals and radioactivity, successional stages, and phytophysiognomies, make bryophytes extremely useful tools for environmental monitoring, restoration, and conservation (Gradstein et al. 2001; Frahm 2003; Vanderpoorten \& Goffinet 2009; Santos et al. 2014; Glime 2017a).

The establishment of protected areas has generally been based on the presence of representative and conspicuous taxa such as vertebrates and vascular plants - leaving bryophyte conservation as almost incidental (Vanderpoorten \& Goffinet 2009), as in the case with the PEVA, where the bryoflora was only recently investigated (Dewes et al. 2021, in press). The effective protection of the entire spectrum of biodiversity will, however, depend on detailed information concerning all taxonomic groups (Oliveira et al. 2017), and the singularities demonstrated by the UPGMA and CA analyses indicated the importance of a detailed knowledge of each part of the Atlantic Forest remnant in the PEVA. Additional studies related to environmental filters and functional groups should contribute greatly to our knowledge of the spatial and temporal patterns of bryophyte composition and diversity in the Atlantic Forest.

\section{Acknowledgements}

We owe special thanks to the Divisão de Unidades de Conservação - Secretaria Estadual do Ambiente e Desenvolvimento Sustentável for granting us the collection license ( ${ }^{\circ}$ 28/2018). The authors thank the administrators of the Parque Estadual de Itapeva for their reception and helpfulness and for allowing collections in all of the phytophysiognomies in the reserve.

\section{References}

Aires T, Garcia M, Bordin J. 2020. Brioflora associada a arroio rural no município de Morro Redondo, Rio Grande do Sul, com novas ocorrências para o Pampa. Pesquisas Botânica 74: 303-323.

Alvarenga LDP, Pôrto KC, Oliveira JRDPM. 2010. Habitat loss effects on spatial distribution of non-vascular epiphytes in a Brazilian Atlantic forest. Biodiversity and Conservation 19: 619-635.

Bates JW. 2009. Mineral nutrition and substratum ecology. In: Goffinet B, Shaw AJ. (eds.) Bryophyte Biology. 2nd. edn. Cambridge, Cambridge University Press. p. 299-356.

Batista WVSM, Pôrto KC, Santos NDD. 2018. Distribution, ecology, and reproduction of bryophytes in a humid enclave in the semiarid region of northeastern Brazil. Acta Botanica Brasilica 32: 303-313. 
Bordin J, Dewes TS, Peralta DF, Ferri M, Rosa BR. 2020. New occurrences of bryophytes species in Southern Brazil: bryodiversity still scarcely known. Check List 16: 915-926.

Bordin J, Yano O. 2009a. Novas ocorrências de antóceros e hepáticas para o Estado do Rio Grande do Sul, Brasil. Brazilian Journal of Botany 32: 189-211.

Bordin J, Yano O. 2009b. Novas ocorrências de musgos (Bryophyta) para o estado do Rio Grande do Sul, Brasil. Brazilian Journal of Botany 32: $455-477$.

Brack P. 2009. Vegetação e paisagem do Litoral Norte do Rio Grande do Sul: exuberância, raridade e ameaças à biodiversidade. In: Würdig NL, Freitas SMF. (eds.) Ecossistemas e biodiversidade do Litoral Norte do RS. Porto Alegre, Nova Prova. p. 32-55.

Buchmann FSC, Caron F, Lopes RP, Ugri A, Lima LGL. 2009. Panorama geológico da Planície Costeira do Rio Grande do Sul. In: Ribeiro AM, Bauermann SG, Scherer CS. (eds.) Quaternário do Rio Grande do Sul: integrando conhecimentos. Porto Alegre, Sociedade Brasileira de Paleontologia. p. 35-56.

Cerqueira GR, Ilkiu-Borges AL, Ferreira LV. 2017. Species richness and composition of epiphytic bryophytes in flooded forests of Caxiuanã National Forest, Eastern Amazon, Brazil. Anais da Academia Brasileira de Ciências 89: 2371-2382.

Clymo RS, Hayward PM. 1982. The ecology of Sphagnum. In: Smith AJE. (ed.) Bryophyte Ecology. London, Chapman and Hall. p. 229-289.

Condit R, Pitman N, Leigh JEG, et al. 2002. Beta-diversity in tropical forest trees. Science 295: 666-669.

Costa DP, Peralta DF. 2015. Bryophytes diversity in Brazil. Rodriguésia 66: 1063-1071.

Costa DP, Pôrto KC, Luizi-ponzo AP, et al. 2011. Synopsis of the Brazilian moss flora: checklist, distribution and conservation. Nova Hedwigia 93: 277334.

Costa DP. 2010. Briófitas. In: Forzza RC, Baumgratz JF, Bicudo CEM, et al. (eds.) Catálogo de plantas e fungos do Brasil. Vol. 1. 1st. edn. Rio de Janeiro, Instituto de Pesquisas Jardim Botânico do Rio de Janeiro. p. 452-521.

Crandall-Stotler B, Stotler RE, Long DG. 2009. Morphology and classification of the Marchantiophyta. In: Goffinet B, Shaw AJ. (eds.) Bryophyte Biology. 2nd. edn. Cambridge, Cambridge University Press. p. 1-54.

Dewes TS, Bordin J, Peralta DF. 2021. As 100 primeiras espécies de briófitas, com sete novos registros para o Sul do Brasil identificadas no Parque Estadual de Itapeva, Torres, Estado do Rio Grande do Sul. Hoehnea (in press).

Duarte MM, Bencke GA. 2006. Plano de Manejo do Parque Estadual de Itapeva: Relatório final. Porto Alegre, Fundação Zoobotânica do Rio Grande do Sul.

Ferraro LMW, Hasenack H. 2009. Clima. In: Würdig NL, Freitas SMF. (eds.) Ecossistemas e Biodiversidade do Litoral Norte do RS. Porto Alegre, Nova Prova. p. 26-31.

Filgueiras TS, Brochado AL, Nogueira PE, Guala GF. 1994. Caminhamento: um método expedito para levantamentos florísticos qualitativos. Cadernos de Geociências 12: 39-43.

Frahm JP. 1991. Dicranaceae: Campylopodioideae, Paraleucobryoideae. Flora Neotropica 54: 1-238.

Frahm JP. 2003. Manual of Tropical Bryology. Tropical Bryology 23: 1-196.

Fudali E. 2001. The ecological structure of the bryoflora of Wroclaw's parks and cemeteries in relation to their localization and origin. Acta Societatis Botanicorum Poloniae 70: 229-235.

Gams H. 1932. Bryocoenology (moss-societies). In: Verdoorn Fr. (ed.) Manual of Bryology. Leiden, Martinus Nijhoff Publishers, The Hague. p. 323-366.

Germano SR, Pôrto KC. 2006. Comunidades de briófitas no remanescente de Mata Atlântica, estado de Pernambuco, Brasil. CryptogamieMartinus Nijhoff Publishers Bryologie 27: 153-163.

Gimingham CH, Birse EM. 1957. Ecological studies on growth-form in bryophytes: I. Correlations between growth-form and habitat. The Journal of Ecology 45: 533-545.

Glime JM. 2017a. Introduction. In: Glime JM. (ed.) Bryophyte Ecology. Vol. 1. Physiological Ecology. https://digitalcommons.mtu.edu/bryophyteecology/. 18 Jan. 2020.
Glime JM. 2017b. Adaptive Strategies: Growth and Life Forms. In: Glime JM. (ed.) Bryophyte Ecology. Vol. 1. Physiological Ecology. https:// digitalcommons.mtu.edu/bryophyte-ecology/. 18 Jan. 2020.

Glime JM. 2017c. Adaptive Strategies: Speculations on Gametophyte Structures. In: Glime JM. (ed.) Bryophyte Ecology. Vol. 1. Physiological Ecology. https://digitalcommons.mtu.edu/bryophyte-ecology/. 18 Jan. 2020.

Glime JM. 2017d. Water Relations: Leaf Strategies - Structural. In: Glime JM. (ed.) Bryophyte Ecology. Vol. 1. Physiological Ecology. https:// digitalcommons.mtu.edu/bryophyte-ecology/. 18 Jan. 2020.

Goffinet B, Buck WR, Shaw AJ. 2009. Morphology, anatomy and classification of the Bryophyta. In: Goffinet B, Shaw AJ. (eds.) Bryophyte Biology. 2nd. edn. Cambridge, Cambridge University Press. p. 55- 138.

Gradstein SR, Churchill SP, Salazar-Allen N. 2001. Guide to the bryophytes of tropical America. Memoirs of the New York Botanical Garden 86: 1-577.

Gradstein SR, Costa DP. 2003. The hepaticae and anthocerotae of Brazil. Memoirs of the New York Botanical Garden 87: 1-318. https://www.researchgate. net/profile/S-Gradstein/publication/248393945_The_Hepaticae_ and_Anthocerotae_of_Brazil/links/59a86f11458515eafa883ad2/TheHepaticae-and-Anthocerotae-of-Brazil.pdf.

Gradstein SR, Pócs T. 1989. Bryophytes. In: Lieth H, Werger MAJ. (eds.) Tropical Rain Forest Ecosystems. Vol. 16. Amsterdam, Elsevier Science Publishers. p. 311-325.

Hedenäs L. 2007. Global diversity patterns among pleurocarpous mosses. The Bryologist 110: 319-331.

Heidtmann LP, Peralta DF, Giroldo D, Hefler SM. 2013. New records of bryophytes for the state of Rio Grande do Sul, Brazil. Acta Botanica Brasilica 27: 626-628.

IBGE - Instituto Brasileiro de Geografia e Estatística. 2012. Manual Técnico da Vegetação Brasileira: Série Manuais Técnicos em Geociências. 2nd. edn. Rio de Janeiro, Instituto Brasileiro de Geografia e Estatística IBGE. https://biblioteca.ibge.gov.br/visualizacao/livros/liv63011.pdf.

IBGE - Instituto Brasileiro de Geografia e Estatística. 2019. Biomas e Sistema Costeiro Marinho do Brasil Compatível com a escala 1:250 000: Série Relatórios Metodológicos. Rio de Janeiro, Instituto Brasileiro de Geografia e Estatística - IBGE. https://biblioteca.ibge.gov.br/ visualizacao/livros/liv101676.pdf.

Koch EW, Barbier EB, Silliman BR, et al. 2009. Non-linearity in ecosystem services: temporal and spatial variability in coastal protection. Frontiers in Ecology and the Environment 7: 29-37.

Löbs N, Walter D, Barbosa CGG, et al. 2019. Microclimatic and ecophysiological conditions experienced by epiphytic bryophytes in an Amazonian rain forest. Biogeosciences Discussions 15. doi: 10.5194/bg-2018-521

Mägdefrau K. 1982. Life-forms of bryophytes. In: Smith AJE. (ed.) Bryophyte Ecology. London, Chapman and Hall. p. 45-58.

Mancino LC, Overbeck GE, Baptista LRM. 2015. Structure of the tree stratum of three swamp forest communities in southern Brazil under different soil conditions. Acta Botanica Brasilica 29: 30-39.

Martins R, Jarenkow JA, Giehl ELH, Citadini-Zanette V, Santos R. 2013. Estrutura de uma floresta brejosa em substrato turfoso, Sul de Santa Catarina, Brasil. Revista Árvore 37: 299-309.

McCune B, Mefford MJ. 1999. PC-ORD: multivariate analysis of ecological data, version 4.10. Gleneden Beach, MjMSofware Design.

Mittermeier RA, Robles-Gil PR, Hoffmann M, et al. 2004. Hotspots revisited: Earth's biologically richest and most endangered terrestrial ecoregions. Washington, CEMEX.

Möls T, Vellak K, Vellak A, Ingerpuu N. 2013. Global gradients in moss and vascular plant diversity. Biodiversity and Conservation 22: 1537-1551.

Mutke J, Barthlott W. 2005. Patterns of vascular plant diversity at continental to global scales. Biologiske Skrifter 55: 521-73.

Oliveira HC, Oliveira SM. 2016. Vertical distribution of epiphytic bryophytes in Atlantic Forest fragments in northeastern Brazil. Acta Botanica Brasilica 30: 609-617.

Oliveira JR, Pôrto KC, Silva MP. 2011. Richness preservation in a fragmented landscape: a study of epiphytic bryophytes in an Atlantic forest remnant in Northeast Brazil. Journal of Bryology 33: 279-290. 


\section{What does a phytophysiognomic mosaic reveal about mosses and liverworts from the subtropical Atlantic Forest?}

Oliveira U, Soares-Filho BS, Paglia AP, et al. 2017. Biodiversity conservation gaps in the Brazilian protected areas. Scientific Reports 7: 1-9.

Pócs T. 1982. Tropical Forest Bryophytes. In: Smith AJE. Bryophyte Ecology. London, Chapman and Hall. p. 59-103.

Proctor MCF. 1979. Structure and ecophysiological adaptation in bryophytes. In: Clarke GCS, Duckett JG. (eds.) Bryophyte systematics. London, Academic Press. p. 479- 509.

Richards PW. 1984. The ecology of tropical forest bryophytes. In: Schuster RM. (ed.) New manual of bryology. Nichinan, Hattori Botanical Laboratory. p. 1233-1270.

Rio Grande do Sul. 2002. Decreto Estadual no 42.009, de 12 de dezembro 2002. Cria o Parque Estadual de Itapeva e dá outras providências. http://www.al.rs.gov.br/legis/M010/M0100099. ASP?Hid_Tipo=TEXTO\&Hid_TodasNormas $=724 \& \mathrm{hTexto}=\& H i d$ IDNorma $=724.01 \mathrm{dez} .2020$.

Santos NDD, Costa DPD, Kinoshita LS, Shepherd GJ. 2011. Aspectos brioflorísticos e fitogeográficos de duas formações costeiras de Floresta Atlântica da Serra do Mar, Ubatuba/SP, Brasil. Biota Neotropica 11: 425-438.

Santos NDD, Costa DPD, Kinoshita LS, Shepherd GJ. 2014. Windborne: can liverworts be used as indicators of altitudinal gradient in the Brazilian Atlantic Forest? Ecology Indicators 36: 431-440.

Santos-Junior R, Silva JG, Martins R. 2015. Estrutura da comunidade arbórea em uma floresta paludosa de restinga na planície costeira do sul do Brasil. Revista Brasileira de Biociências 13: 55-63.

Scott GAM. 1982. Deserts Bryophytes. In: Smith AJE. (ed.) Bryophyte Ecology. London, Chapman and Hall. p. 105-122.

Shaw AJ, Cox CJ, Goffinet B. 2005. Global patterns of moss diversity: taxonomic and molecular inferences. Taxon 54: 337-352.

Shepherd GJ. 2010. FITOPAC 2.1. Versão 2.1. Campinas, SP, Departamento de Botânica, Universidade Estadual de Campinas - UNICAMP.

Silva AC, Berg EVD, Higuchi P, Oliveira Filho AT. 2007. Comparação florística de florestas inundáveis das regiões Sudeste e Sul do Brasil. Brazilian Journal of Botany 30: 257-269.
Silva MPP, Pôrto KC. 2009. Effect of Fragmentation on the Community Structure of Epixylic Bryophytes in Atlantic Forest Remnants in the Northeast of Brazil. Biodiversity and Conservation 18: 317-37.

Silva MPP, Pôrto KC. 2010. Spatial structure of bryophyte communities along an edge-interior gradient in an Atlantic Forest remnant in Northeast Brazil. Journal of Bryology 32: 101-112.

Silva MPP, Pôrto KC. 2013. Bryophyte communities along horizontal and vertical gradients in a human-modified Atlantic Forest remnant. Botany 91: 155-166.

Souza JPS, Silva MPP, Pôrto KC. 2020. Spatial distribution of functional traits of bryophytes along environmental gradients in an Atlantic Forest remnant in north-eastern Brazil. Plant Ecology \& Diversity 13: 93-104.

Sztutman M, Rodrigues RR. 2002. O mosaico vegetacional numa área de floresta contínua da planície litorânea, Parque Estadual da Campina do Encantado, Pariquera-Açu, SP. Brazilian Journal of Botany 25: 161-176.

Takashima-Oliveira TTG, Medeiros PS, Tavares-Martins ACC. 2020. Bryophyte communities across the ecological succession process in the Caxiuanã National Forest, Pará, Brazil. Anais da Academia Brasileira de Ciências 92: e20180626. doi: 10.1590/0001-3765202020180626

Vanderpoorten A, Goffinet B. 2009. Introduction to bryophytes. Cambridge, Cambridge University Press.

Waechter JL. 1985. Aspectos ecológicos da vegetação da restinga no Rio Grande do Sul, Brasil. Comum. Comunicações do Museu de Ciências da PUCRS, série Botânica 33: 49-68.

Weber DA, Bordin J, Prado JF. 2015. Briófitas de um fragmento de mata de restinga do Litoral Norte do Rio Grande do Sul, Brasil. Pesquisas Botânica 67: 81-8.

Worm B, Barbier EB, Beaumont N. et al. 2006. Impacts of biodiversity loss on ocean ecosystem services. Science 314: 787-790.

Záchia RA, Waechter J. 2011. Diferenciação especial de comunidades herbáceo-arbustivas em Florestas Costeiras do Parque Nacional da Lagoa do Peixe, Rio Grande do Sul. Pesquisas, Botânica 62: 211-238. 